\title{
Hemorragia Uterina Disfuncional en el Instituto Materno Infantil 1987-1988
}

\author{
Dres.: Astrid Sánchez *, Jacinto Sánchez A. **, \\ William Onatra H. ${ }^{* * *}$, Ricardo Alvarado P. ****
}

La hemorragia uterina disfuncional entendida como una hemorragia acíclica generalmente anovulatoria sin compromiso orgánico aparece en dos momentos en la vida de la mujer. Dos o tres años después de la menarquia y unos dos a tres años previos a la menopausia. (1). Durante la vida reproductiva la Hemorragia Uterina Disfuncional (HUD) puede presentarse en dos modalidades: ovulatoria y anovulatoria.

La HUD es una entidad que compromete al $50 \%$ de las mujeres entre los 40 y 50 años y en un $20 \%$ de las adolescentes (2). Su mecanismo de acción no está muy bien aclarado pero la mayoría de los investigadores están de acuerdo con que se encuentra asociada a períodos anovulatorios con alteraciones endometriales secundarias a disfunción endocrina del eje hipotálamo -hipófisis- ovario y que estos cambios varían de acuerdo también con la edad en que se encuentre la mujer.

Durante la adolescencia se postula que la HUD obedece a una inmadurez del eje a nivel hipotalámico donde Winter y colaboradores (3) demostraron como los patrones de FSH, LH, Estradiol y Progesterona se encontraban a nivel premenarquial y presentaban una respuesta exagerada de $\mathrm{FSH}$ a la administración de GnRH. Por otra parte Fraser y col. (4), encontraron que los patrones de Estrona y Estradiol estaban dentro de límites normales pero el pico de LH que debían ejecutar los estrógenos como mecanismo de retroalimentación positiva estaba ausente o disminuido, demostrando con esto que durante la adolescencia los ciclos son anovulatorios por no respuesta a nivel del SNC.

\footnotetext{
* Estudiante postgrado. III año de Ginecología y Obstetricia.

** Instructor Asociado Dpto. Ginecología y Obstetricia.

*** Profesor Asistente Dpto. Ginecología y Obstetricia.

**** Profesor Asociado Dpto. Patología. Facultad de Medicina. Universidad Nacional de Colombia.
}

En la vida reproductiva la HUD se ha dividido en Anovulatoria y Ovulatoria. En la primera se demuestra una alteración en el mecanismo de retroalimentación con fluctuaciones en la LH, Estradiol y Andrógenos ováricos. Se postula además que puede existir una anormal conversión periférica de andrógenos a estrógenos y un defecto en la liberación de prostaglandinas a nivel endometrial (5). Por otra parte se ha demostrado que en el líquido folicular se encuentra un aumento en la concentración de Estrona (E1) y Estradiol (E2) al compararlo con mujeres normales, observación que demuestra una alteración no solo de ganadotrofinas sino de los esteroides a nivel ovárico (2).

En general, podemos decir que la HUD obedece a una alteración en el mecanismo de retroalimentación por alteración en la concentración de Estrógenos y una nula o poca producción de progesterona. Como consecuencia de esto se presenta una proliferación del endometrio persistente. Esta proliferación exagerada lleva a una disminución de la circulación y por lo tanto a una baja de los nutrientes como los polisacáridos. Este desarrollo anormal hace que las glándulas estromales se deformen con liberación de enzimas lisosomales que pueden contribuir al sangrado irregular que presentan estas pacientes. (5). A pesar de que no se ha demostrado que los Estrógenos por sí aumenten la actividad fibrinolítica, esta aparece alterada haciendo que persista la hemorragia.

La HUD de tipo ovulatorio se encuentra asociada a defectos del Cuerpo Lúteo como sucede en la Enfermedad de Halban, -Persistencia del cuerpo lúteo-; Insuficiencia de Cuerpo Lúteo o inadecuada producción de progesterona; en una entidad recientemente reportada como No Ruptura Folicular (6), donde no hay expulsión del óvulo, pero la luteinización se lleva a cabo con niveles disminuidos de progesterona, estimulando pobremente el endometrio. 
Durante la perimenopausia es bien conocido que los ciclos se hacen irregulares, generalmente anovulatorios y su mecanismo de acción obedece a una progresiva refractariedad del ovario a las Gonadotrofinas (7). Cuando una hemorragia persiste o aparece después de la menopausia se hace imperioso descartar algún proceso neoplásico por medio de la biopsia endometrial.

\section{MATERIAL Y METODOS}

Se realizó un estudio descriptivo de tipo retrospectivo en el Departamento de Obstetricia y Ginecología de la Facultad de Medicina de la Universidad Nacional de Colombia, desde el 1o. de marzo de 1986 al 28 de febrero de 1987, en pacientes que consultaron al servicio de Urgencias (Admisión) del Instituto Materno Infantil de Bogotá y que fueron diagnósticas clínicamente como Hemorragia uterina disfuncional (HUD).

A estas pacientes se les elaboró Historia Clínica (HC), solicitándoles Hemoclasificación, Serología, cuadro Hemático y VSG, Glicemia. En las mayores de 45 años con antecedentes de hipertensión electrocardiograma y valoración cardiológica. Fueron llevadas a legrado biopsia bajo anestesia general y los hallazgos del examen consignados en la H.C. El material obtenido se colocó en formol al $10 \%$ y enviado al Departamento de Anatomía Patológica donde se analizaron las muestras con los criterios de Noyes y Novack (8). Las pacientes fueron remitidas a la consulta externa donde de acuerdo con estudio histopatológico se realizó el manejo correspondiente

Fue interés de los autores conocer la frecuencia de la HUD en un hospital de tercer nivel; la patología que pudiera corresponder a este síntoma, su relación con la edad, paridad y planificación familiar. Evaluar

\section{GRAFICA}

\section{FRECUENCIA DE LA H.U.D.}

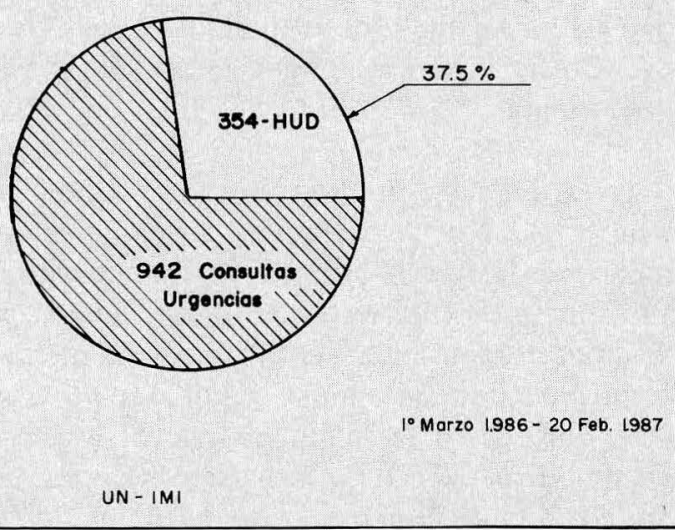

GRAFICA 2

DIAGNOSTICO DE LA H.U.D.

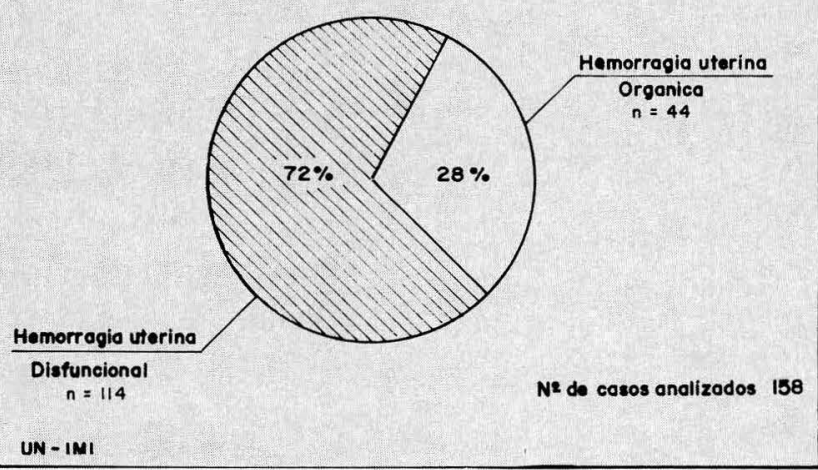

el manejo que se estaba dando a esta entidad y de acuerdo con los resultados proponer una norma de orientación para una entidad frecuente en Medicina general y Ginecología.

\section{RESULTADOS}

Durante el período del 1o. de marzo de 1986 y el 28 de febrero de 1987, en el servicio de Urgencias del Instituto Materno Infantil se presentaron 942 consultas ginecológicas de las cuales 354 (37.5\%) fueron diagnósticas como HUD. De estas se tabularon 158 pacientes y después de haber obtenido el resultado de Anatomía Patológica se encontró que 114 (72\%) correspondían a HUD y $44(28 \%)$ a hemorragia uterina de tipo orgánico. (HUO).

La distribución según los grupos etarios mostró los siguientes resultados: Tabla No. 1. Gráfica No. 3.

GRAFICA 3

\section{DISTRIBUCION POR EDAD DE LA H.U.D}

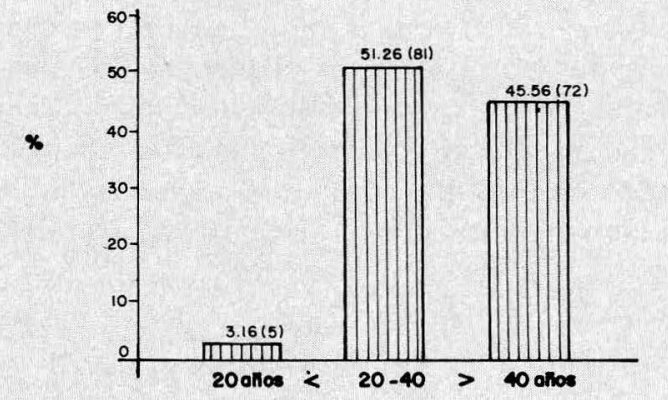


TABLA 1

EDAD Y TIPO DE HEMORRAGIA

\begin{tabular}{|crrrrrrr|}
\hline Grupos de Edad & & HUD & \multicolumn{2}{c|}{ HUO } & \multicolumn{2}{c|}{ Total } \\
\hline años & n & \% & n & \% & n & $\%$ \\
\hline $15-19$ & 4 & 3.5 & 1 & 2.3 & 5 & 3.2 \\
$20-29$ & 32 & 22.2 & 14 & 31.8 & 46 & 29.1 \\
$30-39$ & 31 & 27.2 & 5 & 11.4 & 36 & 22.8 \\
$40-49$ & 37 & 32.4 & 18 & 40.9 & 55 & 34.8 \\
$50-59$ & 10 & 8.7 & 6 & 13.6 & 16 & 10.1 \\
60 & - & - & - & - & - & - \\
Total & 114 & 100.0 & 44 & 100.0 & 158 & 100.0 \\
\hline
\end{tabular}

\section{GRAFICA 4}

\section{DISTRIBUCION POR EDAD/H.U.D. / H.U.O.}

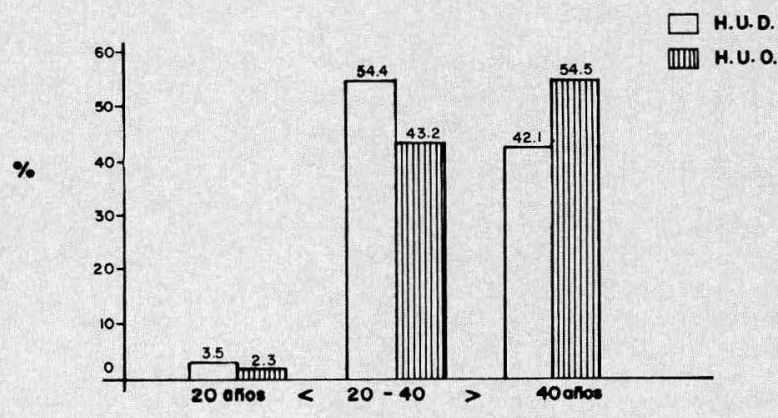

UN - IM

Con el fin de tener una visión más objetiva del comportamiento de la HUD frente a la edad decidimos clasificarla en 3 grandes grupos: I menores de 20 años; II entre 20 y 40 años y III mayores de 40 años. Gráfica No. 4

Al analizar los estudios de Histopatología para la hemorragia Uterina Disfuncional y la Orgánica encontramos los siguientes resultados: Tabla No. 2. Gráfica No. 5

TABLA 2

GRUPOS DE EDAD / TIPO DE ENDOMETRIO

\begin{tabular}{|c|c|c|c|c|c|c|c|c|}
\hline \multirow{2}{*}{$\begin{array}{l}\text { Grupos de } \\
\text { Edad }\end{array}$} & \multicolumn{2}{|c|}{ E. Secretor } & \multicolumn{2}{|c|}{ E. Proliferativo } & \multicolumn{2}{|c|}{ E. Atrófico } & \multicolumn{2}{|c|}{ Total } \\
\hline & $\mathrm{n}$ & $\%$ & $\mathbf{n}$ & $\%$ & n & $\%$ & & $\%$ \\
\hline 2 & 2 & 1.7 & 2 & 1.7 & & & 4 & 3.5 \\
\hline $20-40$ & 27 & 23.6 & 33 & 28.9 & $2(\mathrm{MI})$ & 1.7 & 62 & 54.4 \\
\hline 40 & 14 & 12.3 & 32 & 28.0 & $2(\mathrm{~A})$ & 1.7 & 48 & 42.1 \\
\hline Total & 43 & 37.7 & 67 & 58.8 & 4 & 3.5 & 114 & 100.0 \\
\hline
\end{tabular}

MI. = Material Insuficiente. $\mathrm{A}=$ Aborto
GRAFICA 5

\section{DISTRIBUCION POR EDAD/TIPO ENDOMETRIO EN LA HEMORRAGIA UTERINA DISFUNCIONAL}

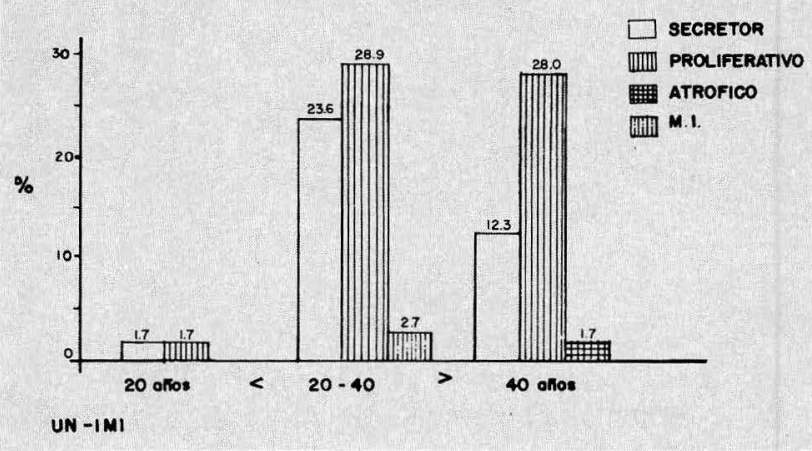

De acuerdo con el método de planificación familiar quisimos saber como se comportaba la HUD.Los resultados aparecen en la siguiente Tabla No. 4

En relación con el manejo y control de este grupo de pacientes encontramos que de las 67 pacientes con E/Proliferativo $47(70.1 \%)$ fueron controladas 37 (78.7) de las cuales mostraron mejoría 20 (54.0\%), 15 (40.5) requirieron tratamiento con Medroxiprogesterona por 3 meses y $2(5.4 \%)$ histerectomía con ID de miomatosis uterina. Las 10 restantes $(21.3 \%)$ no volvieron a control. De las 43 pacientes con E. Secretor 38 (88.3\%) mostraron mejoría después del legrado; 5 (11.6) se complementó el tratamiento con anovulatorios por deseo de planificar.

En la Hemorragia Uterina Orgánica, para los casos de endometritis se inició terapéutica con antibióticos y control posterior para descartar procesos TBC. En la hiperplasia endometrial $4(30.7)$ recibieron tratamiento con Medroxiprogesterona y $6(46.15 \%)$ se les practicó histerectomía abdominal con $\mathrm{SOB}$ ante el riesgo de $\mathrm{Ca}$. endometrial. $3(23.0 \%)$ no regresaron a control.

GRAFICA 6

DISTRIBUCION POR EDAD / TIPO ENDOMETRIO EN LA HEMORRAGIA UTERINA ORGANICA

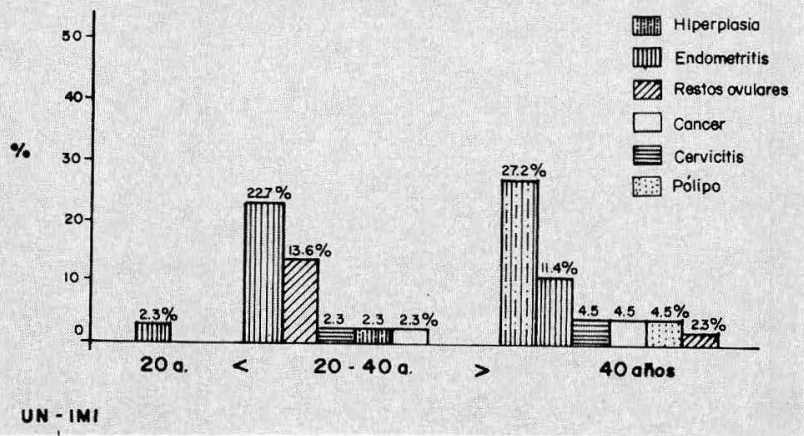


TABLA 3

GRUPOS DE EDAD. TIPO DE ENDOMETRIO HUO

\begin{tabular}{|c|c|c|c|c|c|c|c|c|c|c|c|c|c|c|}
\hline \multirow{2}{*}{$\begin{array}{c}\text { Grupos de } \\
\text { Edad }\end{array}$} & \multicolumn{2}{|c|}{ E. tritis } & \multicolumn{2}{|c|}{ R. Ovul. } & \multicolumn{2}{|c|}{ C.C. } & \multicolumn{2}{|c|}{ H/Plasia } & \multicolumn{3}{|c|}{ Pólipo Ca. } & \multirow[b]{2}{*}{$\%$} & \multicolumn{2}{|c|}{ Total } \\
\hline & n & & n & $\%$ & n & $\%$ & n & $\%$ & $\mathbf{n}$ & $\%$ & n & & n & $\%$ \\
\hline 20 & 1 & 2.3 & & & & & & & & & & & 1 & 2.3 \\
\hline $20-40$ & 10 & 22.7 & 6 & 13.6 & 1 & 2.3 & 1 & 2.3 & & & 1 & 2.3 & 19 & 43.2 \\
\hline 40 & 5 & 11.4 & 1 & 2.3 & 2 & 4.5 & 12 & 27.3 & 2 & 4.5 & 2 & 4.5 & 24 & 54.5 \\
\hline Total & 16 & 36.4 & 7 & 15.9 & 3 & 6.8 & 13 & 29.3 & 2 & 4.5 & 3 & 6.8 & 44 & 100.0 \\
\hline
\end{tabular}

\section{DISCUSION}

La frecuencia de la HUD afecta entre un 10 y $35 \%$ de todas las pacientes ginecológicas y constituye un $20-25 \%$ de todas las operaciones ginecológicas (1). En un análisis previo realizado por la Dras. Fonnegra L y Rojas L (9) en el IMI en 1984 encuentran que la frecuencia fue del $1.66 \%$ de todas las urgencias ginecobstétricas. Jones Smith y Jones HW (10) muestran que la frecuencia fue de $35 \%$ y $15 \%$ de acuerdo con nuestros resultados $(37.5 \%)$ si bien muestra una mayor frecuencia se debe tener en cuenta que los datos están tomados de un hospital de referencia.

Diferentes autores están de acuerdo con clasificar para efectos prácticos en los 3 grupos: adolescencia, vida reproductiva y menopausia. La frecuencia promedio muestra que es del 20,30 y $50 \%$ respectivamente. Nuestros datos, a pesar del número escaso, por debajo de los 20 años (3.5) y por no ser una hospital pediátrico es posible que esta frecuencia sea menor, pero durante la vida reproductiva y perimenopáusica las cifras no difieren de lo reportado en la literatura (55.4 y 42.1).

Llama la atención que de las pacientes catalogadas al ingreso como HUD, después de los estudios de histopatología, sólo el $72 \%$ correlacionó con esta entidad y el $28 \%$ a una causa orgánica. En una investigación hecha por Claessen (12) durante la adolescencia encuentra que la HUD de tipo disfuncional fue del $74 \%$ y la orgánica de $24 \%$ mientras que Czernobilsky (13) tomando 100 biopsias endometriales de una población general encuentra $83 \%$ de HUD y $17 \%$ de HUO.

TABLA 4

PLANIFICACION . HUD - HUO

\begin{tabular}{|lrrrrrr|}
\hline Tipo de Planificación & \multicolumn{2}{c}{ HUD } & \multicolumn{2}{c|}{ HUO } & \multicolumn{2}{c|}{ Total } \\
& n & \% & \multicolumn{1}{c|}{ n } & \% & \multicolumn{1}{c|}{ n } & \% \\
\hline Anovulatorios & 12 & 23.0 & 7 & 13.5 & 19 & 36.5 \\
Dispositivo(DIU) & 13 & 25.0 & 6 & 11.5 & 19 & 36.5 \\
Ligadura (Pomeroy) & 12 & 23.0 & 2 & 3.8 & 14 & 26.9 \\
Total & 37 & 71.1 & 15 & 28.8 & 52 & 100.0 \\
\hline
\end{tabular}

GRAFICA 7

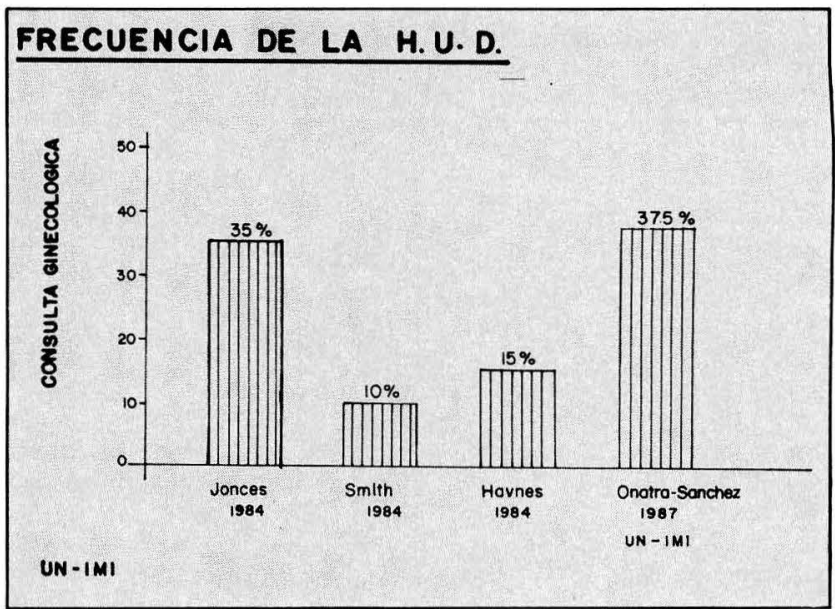

De acuerdo con la fisiopatología, se postula que los ciclos son anovulatorios. Nuestro estudio demuestra que esto no es totalmente cierto. A pesar del número escaso durante la adolescencia el endometrio secretor como el proliferativo guardan la proporción del $1.7 \%$. En las pacientes por encima de los 40 años hay un predominio del E. Proliferativo sobre el secretor $(28.0 \% / 12.3 \%)$ como era de esperarse. Durante la vida reproductiva, contrario a la descripción de la literatura (10\%), hay un equilibrio entre el E. secretor $23.8 \% /$ E. Proliferativo $28.9 \%$, lo que nos permite afirmar que se deben buscar las causas no sólo anovulatorias sino también las ovulatorias. En este último grupo, el equipo de la Universidad de Antioquia está de acuerdo (14).

En relación con la HUO es importante destacar que todos los tres grupos de edad aparece la endometritis (36.4\%) como un factor constante y que amerita un estudio más profundo sobre el tema pues a pesar del estado socioeconómico de nuestra población no es una explicación satisfactoria para este hecho. Nos pareció de interés encontrar restos ovulares en pacientes de edad que a veces nos confunde con los casos de hiperplasia y donde solo la patología nos hace el diagnóstico. La hiperplasia endometrial es uno de los factores predominantes $29.1 \%$ en las pacientes por encima de los 40 
GRAFICA 8

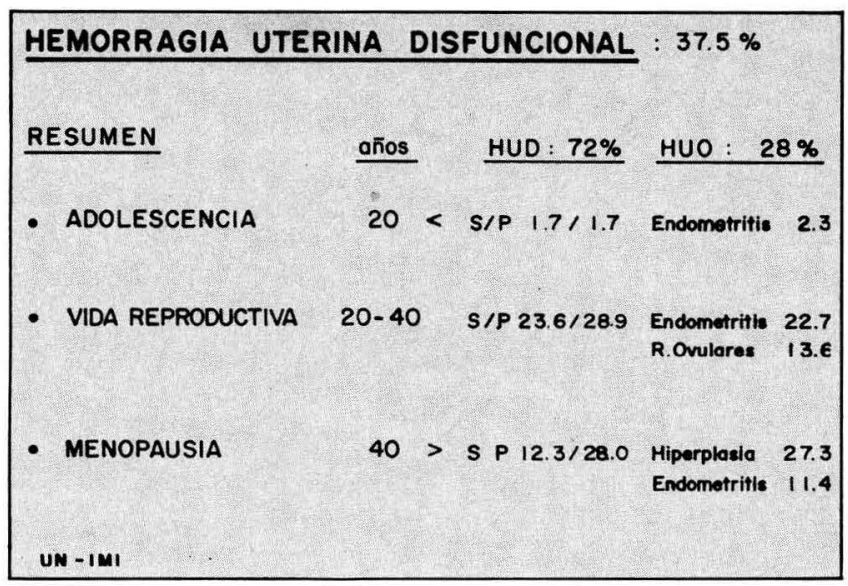

años y llamamos la atención sobre la importancia de practicar el legrado o biopsia de rutina en este grupo. El cáncer endometrial aparece pero su frecuencia tan baja, $6.8 \%$ está de acuerdo para este tipo de estudio (13)(14)(15).

Una hipótesis propuesta por algunos ginecólogos informa sobre los trastornos del ciclo en pacientes sometidas a ligadura o a DIU. Los datos de este estudio no pudieron confirmar esa hipótesis, pues desde el punto de vista estadístico no hubo diferencia en los 3 grupos de planificación familiar.

En relación con el manejo de la HUD no se han modificado las normas establecidas en la mayoría de las Escuelas de Medicina. Una de las fallas en nuestro medio es el seguimiento, en parte atribuido a nivel sociocultural de nuestras pacientes y a la falta de moti- vación por parte del personal de salud. El legrado sigue siendo un buen método diagnóstico para este tipo de sintomatología y amerita profundizar en el estudio de las causas ovulatorias y el clasificar el tipo de hiperplasia nos permitirá un manejo más racional de HUD. Gráfica No. 8.

\section{RESUMEN}

Se analizaron 158 Historias Clínicas en forma retrospectiva de pacientes entre 16 y 60 años con diagnóstico de Hemorragia Uterina Disfuncional (HUD) que ingresaron por el Servicio de Admisión (Urgencias) del Hospital Materno Infantil de Bogotá entre el 1o. de marzo de 1986 y el 30 de septiembre del mismo año. De 942 consultas 354 (37.5\%) correspondieron a HUD. Se analizaron previo estudio histopatológico 158 casos. De estos 114 (72.15\%) fueron de HUD y 44 (27.85\%) a HUO. Al Clasificar por grupos de edad se aprecia que las pacientes menores de 20 años, 5 (3.16\%); entre 20 y 40 años $81(54.26 \%$ ) y mayores de 40 años 72 (45.56\%). Los diagnósticos definitivos para la HUD fueron E. Proliferativo 67 casos (58.8\%); Secretor 43 (37.7\%) y Atrófico $2(1.75 \%)$. Para la HUO: Endometritis 16 casos $(36.4 \%)$ Hiperplasia Endometrial 13(29.6\%); restos ovulares 7(15.9\%); Ca. Endometrial $3(6.8 \%)$ y Pólipos 2 (4.5\%). El tratamiento de legradobiopsia fue eficaz en el 54\% para el E. Proliferativo y $88.3 \%$ para el secretor. La terapéutica hormonal complementaria se llevó a cabo en el $40.5 \%$ de los casos de E. Proliferativo y $11.6 \%$ en E. Secretor. En la HUO, la Endometritis recibió tratamiento terapéutico en el $90 \%$ de los casos y en la hiperplasia $4(30.7 \%)$ y quirúrgico $6(46.1 \%)$. Se recomienda el legrado biopsia en pacientes con trastornos del ciclo para mayores de 40 años.

BIBLIOGRAFIA

1. SPELLACY, W.N. Hemorragias Ultrinas. Clínicas Obstétricas y Ginecológicas. Editorial Interamericana. 3: 853, 1983.

2. MISHELL, D.R.; DAVAJAN, V. Reproductive Endocrinology, Infertility and Contraception. F.A. Davis Company. Philadelphia 1979.

3. WINTER, J.S.D.; FAIMAN, S. The development of cyclic Pituitary-gonadal function in adolescent females. J. Clin. Endocrinol. Metab. 37: 714, 1973.

4. FRASER, I.S. Pituitary gonadotrophins and ovarian function in adolescent dysfunctional uterine bleeding. J. Clin. Endocrinol Metab, 37: 407, 1973.

5. YEN, S.S.C.; JAFFE, R.B. Reproductive Endocrinology. Second Edition/W.B. Saunders company 1986.
6. DALY, C.D.; SOTO-ALBORS, C. Ultrasonographic assessment of luteinized unruptured follicle syndrome in unexplained infertility. Fertil Steril 43: 62, 1985.

7. GREEN, M.F. Geriatric Endocrinology. Clinical Endocrinology. William Heinemann Medical Books. London. 1984.

8. NOYES, R.W.; HERTIG, A.T. Dating the endometrial biopsy. Fertil Steril 1: 3, 1950.

9. ROJAS, L.; FONNEGRA, J. Hemorragia Uterina disfuncional y su resultado Histopatológico. Trabajo de promoción R.. 1984 Archivos Dpto de Obstétricia y Ginecología. U. Nacional de Colombia.

10. JONES, H.W.; JONES, G.S. Tratado de Ginecología de Novack. Edit. Interamericana. 11 Edición. 1988. 
11. HITCHEK, A. Hemorragia uterina disfuncional en la adolescencia. Clin. Obstet. Gynecol. 3: 643, 1977. Interamericana.

12. CLAESSENS, E.A.; COWELL, C.A. Acute adolescent menorrahia. Am. J. Obstet Gynecol 139: 277, 1982.

13. CZERNOBILSKY, B. Utero-ovarian pathology in dysfunctional uterine bleeding. Clin. Obstet. Gynecol. Edit. Harper. Row. 13(2): 416, 1970.
14. BOTERO, J.; JUBIZ, H.; HENAO, G. Obstetricia y Ginecología. Edit. Carvajal. 1985.

15. CHARLES, S.F,; Dysfunctional uterine bleeding Primary Care 15(3): 561, 1988.

16. COLLINS, J.B. Menopause. Primary Care. 15(3): 593, 1988.

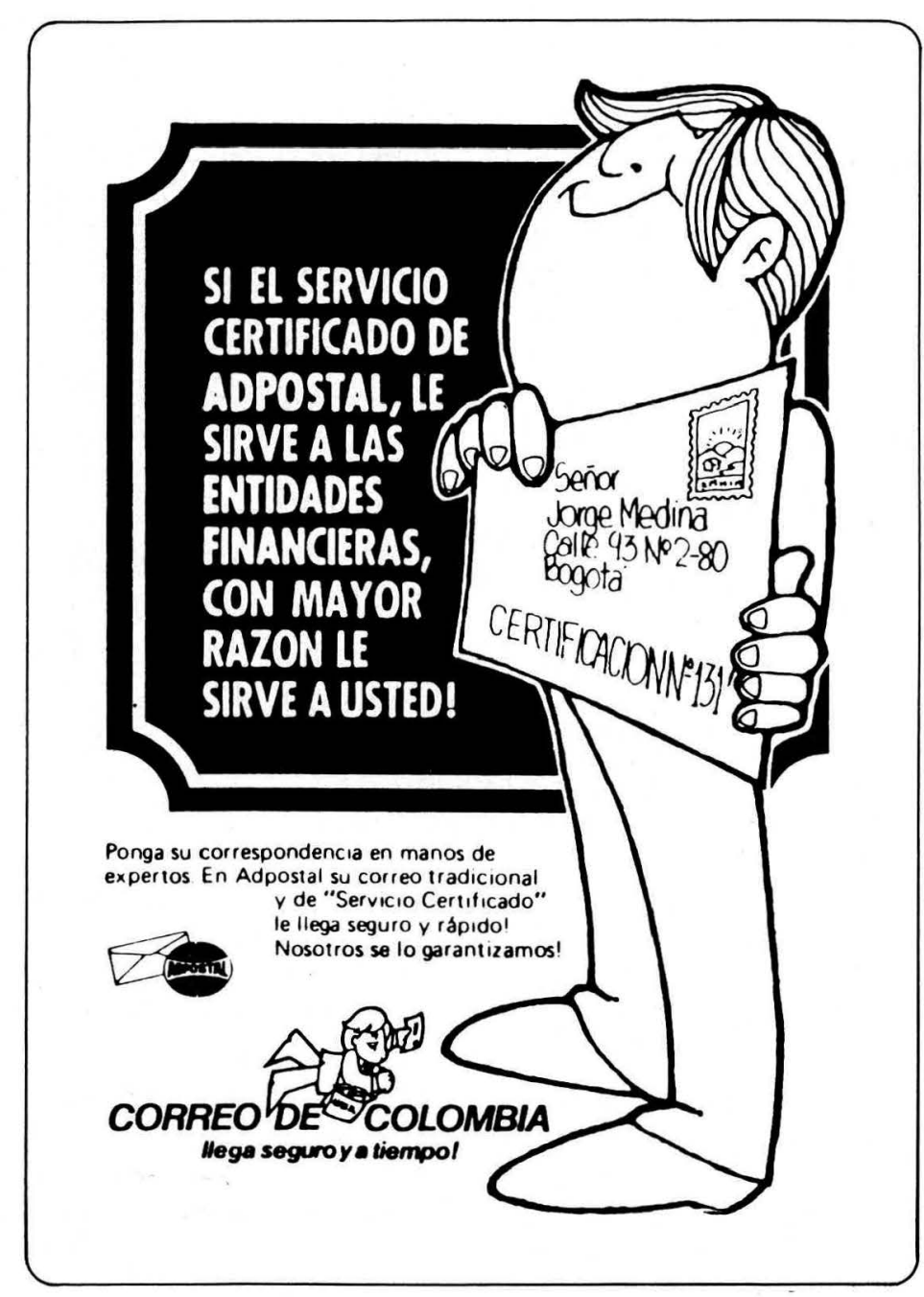

